The $\mathbf{J}_{\text {ournal of }} \mathbf{N}_{\text {onlinear }} \mathbf{S}_{\text {ciences and }}$ A pplications

http://www.tjnsa.com

\title{
NONTRIVIAL SOLUTIONS OF SINGULAR SECOND ORDER THERE-POINT BOUNDARY VALUE PROBLEM AT RESONANCE
}

\author{
XIAORONG WU ${ }^{1}$ AND FENG WANG ${ }^{2 *}$
}

\begin{abstract}
The singular second order three-point boundary value problem at resonance

$$
\left\{\begin{array}{l}
x^{\prime \prime}(t)=f(t, x(t)), \quad 0<t<1, \\
x^{\prime}(0)=0, \quad x(\eta)=x(1),
\end{array}\right.
$$

are considered under some conditions concerning the first eigenvalues corresponding to the relevant linear operators, where $\eta \in(0,1)$ is a constant, $f$ is allowed to be singular at both $t=0$ and $t=1$. The existence results of nontrivial solutions are given by means of the topological degree theory.
\end{abstract}

\section{INTRODUCTION}

This paper is concerned with the existence of at least one nontrivial solution for the nonlinear singular second order three-point boundary value problem at resonance

$$
\left\{\begin{array}{l}
x^{\prime \prime}(t)=f(t, x(t)), \quad 0<t<1 \\
x^{\prime}(0)=0, \quad x(\eta)=x(1)
\end{array}\right.
$$

where $\eta \in(0,1)$ is a constant, $f$ is allowed to be singular at both $t=0$ and $t=1$. The problem (1.1) happens to be at resonance in the sense that the associated linear homogeneous boundary value problem

$$
\left\{\begin{array}{l}
x^{\prime \prime}(t)=0, \quad 0<t<1, \\
x^{\prime}(0)=0, \quad x(\eta)=x(1),
\end{array}\right.
$$

has $x(t)=c, c \in \mathbf{R}$ as a nontrivial solution.

Date: Received: 7 July 2008; Revised: 28 July 2008.

* Corresponding author.

2000 Mathematics Subject Classification. Primary 34B15; Secondary 34B25.

Key words and phrases. Singular, Nontrivial solutions, Boundary value problem, Topology degree, Resonance. 
Multi-point boundary value problem of second order differential equations have been studied intensively and the resonance cases have received more attention [1]-6]. Recently, Ma 2] developed the method of upper and lower solutions for nonlinear three-point boundary value problem at resonance and established some multiplicity results. Bai [3] generalized the existence results to the four-point boundary value problem at resonance by using coincidence degree theory due to Mawhin [7]. Very recently, the existence of positive or nonnegative solutions for multi-point BVP has been studied by several authors, see for example [4]-6] and the references therein. However, few results are available for the existence of nontrivial solutions for singular multi-point boundary value problem at resonance. Motivated by [4], we establish the existence results of nontrivial solutions for the singular boundary value problem (1.1) at resonance by means of the topological degree theory under some conditions concerning the first eigenvalue corresponding to the relevant linear operator. The eigenvalue criteria of this sort for nonlinear two-point boundary value problem is established in [8]. For the concepts and properties about the cone theory and the topological degree we refer to [9]-[11].

\section{PRELIMINARIES}

In this section, we shall give some preliminaries. In the Banach space $C[0,1]$ in which the norm is defined by $\|x\|=\max _{0 \leq t \leq 1}|x(t)|$. We set

$$
P=\{x \in C[0,1] \mid x(t) \geq 0, \quad t \in[0,1]\} .
$$

$P$ is a positive cone in $C[0,1]$. Throughout this section, the partial ordering is always given by $P$. We denote by $B_{r}=\{x \in C[0,1] \mid\|x\|<r\}(r>0)$ the open ball of radius $r$.

Define $g(t, x)=f(t, x)+\beta^{2} x$. For convenience, we make the following assumptions:

(H1) $\beta \in\left(0, \frac{\pi}{2}\right)$ is a constant.

(H2) $f:(0,1) \times(-\infty,+\infty) \rightarrow(-\infty,+\infty)$ is continuous and

$$
h_{1}(t) g_{1}(x) \leq g(t, x) \leq h_{2}(t) g_{2}(x), \quad(t, x) \in(0,1) \times(-\infty,+\infty),
$$

where $h_{1}(t), h_{2}(t) \in C((0,1),[0,+\infty)), g_{1}(x), g_{2}(x) \in C((-\infty,+\infty),(-\infty,+\infty))$, and $h_{1}(t) \not \equiv 0, h_{2}(t) \not \equiv 0, t \in(0,1)$.

$$
\int_{0}^{1} h_{2}(t) d t<+\infty
$$

It is known (see [4] ) that BVP (1.1) is equivalent to the problem

$$
\left\{\begin{array}{l}
x^{\prime \prime}(t)+\beta^{2} x(t)=g(t, x(t)), \quad 0<t<1 \\
x^{\prime}(0)=0, \quad x(\eta)=x(1)
\end{array}\right.
$$

As is well known, the singular nonlinear boundary value problem (1.1) can be converted into the equivalent Hammerstein nonlinear integral equation

$$
x(t)=\int_{0}^{1} G(t, s) g(s, x(s)) d s, \quad t \in[0,1],
$$


where $G(t, s)$ (see [4]) is defined by

$$
\begin{aligned}
G(t, s)= & \begin{cases}\frac{1}{\beta} \sin \beta(t-s), & 0 \leq s \leq t \leq 1, \\
0, & 0 \leq t \leq s \leq 1,\end{cases} \\
& +\frac{\cos \beta t}{\beta \sin \frac{\beta(\eta+1)}{2}} \begin{cases}\cos \frac{\beta(2 s-\eta-1)}{2}, & 0 \leq s \leq \eta<1, \\
\frac{\sin \beta(1-s)}{2 \sin \frac{\beta(1-\eta)}{2}}, & 0<\eta \leq s \leq 1 .\end{cases}
\end{aligned}
$$

Obviously, for any $t, s \in[0,1]$, we have $G(t, s) \geq 0$ by $(H 1)$.

Let

$$
\begin{gathered}
(A x)(t)=\int_{0}^{1} G(t, s) g(s, x(s)) d s, \quad t \in[0,1] . \\
\left(T_{1} x\right)(t)=\int_{0}^{1} G(t, s) h_{1}(s) x(s) d s, \quad t \in[0,1] . \\
\left(T_{2} x\right)(t)=\int_{0}^{1} G(t, s) h_{2}(s) x(s) d s, \quad t \in[0,1] .
\end{gathered}
$$

By the method similar to that in [8], we have

Lemma 2.1. Suppose that $(H 1)-(H 3)$ are satisfied, then $A: C[0,1] \rightarrow C[0,1]$ is a completely continuous operator, $T_{1}, T_{2}: C[0,1] \rightarrow C[0,1]$ are completely continuous linear operators and $T_{1}, T_{2}: P \rightarrow P$.

It is not difficult to verify that the nonzero fixed points of the operator $A$ are nontrivial solutions of singular boundary value problem (1.1).

Lemma 2.2. Suppose that the conditions (H1), (H2) are satisfied, then for the operators $T_{1}, T_{2}$ defined by (2.1), (2.2), the spectral radius $r\left(T_{1}\right), r\left(T_{2}\right) \neq 0$ and $T_{1}, T_{2}$ have the positive eigenfunctions corresponding to their first eigenvalue $\lambda_{1}=$ $\left(r\left(T_{1}\right)\right)^{-1}, \widetilde{\lambda_{1}}=\left(r\left(T_{2}\right)\right)^{-1}$ respectively.

We also need the following lemmas in 9 .

Lemma 2.3. Let $P$ be a cone in a real Banach space $E, \Omega$ a bounded open subset of $E$, and $A: P \cap \bar{\Omega} \rightarrow P$ a completely continuous operator. Assume that there exists a $u_{0} \in P, u_{0} \neq \theta$ such that

$$
x-A x \neq \mu u_{0},
$$

for all $x \in P \cap \partial \Omega$ and $\mu \geq 0$, then the fixed point index

$$
i(A, P \cap \Omega, P)=0 \text {. }
$$

Lemma 2.4. Let $P$ be a cone in a real Banach space $E, \Omega$ a bounded open subset of $E$ with $\theta \in \Omega$, and $A: P \cap \bar{\Omega} \rightarrow P$ a completely continuous operator. If

$$
A x \neq \mu x,
$$

for all $x \in P \cap \partial \Omega$ and $\mu \geq 1$, then the fixed point index

$$
i(A, P \cap \Omega, P)=1 \text {. }
$$




\section{MAin RESUlts}

Theorem 3.1. Suppose that the conditions $(H 1)-(H 3)$ are satisfied. If there exists a constant $b \geq 0$ such that

$$
\begin{gathered}
f(t, x) \geq-\beta^{2} x-b, \quad \forall t \in[0,1], x \in \mathbf{R}, \\
\liminf _{x \rightarrow 0} \frac{g_{1}(x)}{|x|}>\lambda_{1}, \\
\limsup _{x \rightarrow+\infty} \frac{g_{2}(x)}{x}<\widetilde{\lambda_{1},}
\end{gathered}
$$

where $\lambda_{1}, \widetilde{\lambda_{1}}$ are the first eigenvalue of $T_{1}$ and $T_{2}$ respectively. Then the singular boundary value problem (1.1) has at least one nontrivial solution.

Proof. It follows from (3.2) that there exists $r_{1}>0$ such that

$$
g_{1}(x) \geq \lambda_{1}|x|, \quad \forall|x| \leq r_{1} .
$$

For every $x \in \bar{B}_{r_{1}}$, we have from 3.4 that

$$
(A x)(t) \geq \lambda_{1} \int_{0}^{1} G(t, s) h_{1}(s)|x(s)| d s \geq 0, \quad t \in[0,1],
$$

and thus $A\left(\bar{B}_{r_{1}}\right) \subset P$. For any $x \in \partial B_{r_{1}} \cap P$, it follows from (3.4) that

$$
(A x)(t) \geq \lambda_{1} \int_{0}^{1} G(t, s) h_{1}(s) x(s) d s=\lambda_{1}\left(T_{1} x\right)(t), \quad t \in[0,1],
$$

We may suppose that $A$ has no fixed point on $\partial B_{r_{1}}$ (otherwise, the proof completes). Let $x^{*}$ be the positive eigenfunction of $T$ corresponding to $\lambda_{1}$, thus $x^{*}=\lambda_{1} T x^{*}$. As in [8] we can prove that $x-A x \neq \tau x^{*}, \forall x \in \partial B_{r_{1}} \cap P, \tau \geq 0$. Since $A\left(\bar{B}_{r_{1}}\right) \subset P$, we have from the permanence property of fixed point index and Lemma 2.3 that

$$
\operatorname{deg}\left(I-A, B_{r_{1}}, \theta\right)=i\left(A, B_{r_{1}} \cap P, P\right)=0,
$$

where deg denotes the topological degree.

Letting $\widetilde{x}(t)=b \int_{0}^{1} G(t, s) d s$. Obviously, $\widetilde{x} \in P$. It is easy to see from 3.1 that $A: C[0,1] \rightarrow P-\widetilde{x}$. Define $\widetilde{A} x=A(x-\widetilde{x})+\widetilde{x}, x \in C[0,1]$, then $\widetilde{A}:$ $C[0,1] \rightarrow P$.

It follows from (3.3) that there exist $r_{2}>r_{1}+\|\widetilde{x}\|$ and $0<\sigma<1$ such that

$$
g_{2}(x) \leq \sigma \widetilde{\lambda_{1}} x, \quad t \in[0,1], x \geq r_{2} .
$$

Let $\widetilde{T}_{2} x=\sigma \widetilde{\lambda_{1}} T_{2} x, x \in C[0,1]$. Then $\widetilde{T}_{2}: C[0,1] \rightarrow C[0,1]$ is a bounded linear operator and $\widetilde{T}_{2}(P) \subset P$. Let

$$
M=2 \max \left\{\sup _{x \in \bar{B}_{r_{2}}} \int_{0}^{1} G(s, s) h_{2}(s)\left|g_{2}(x(s))\right| d s, 2\|\widetilde{x}\|\right\} .
$$


It is clear that $M<+\infty$. Let

$$
W=\{x \in P \mid x=\mu \widetilde{A} x, 0 \leq \mu \leq 1\} .
$$

In the following, we prove that $W$ is bounded.

For any $x \in W$, set $\widetilde{y}(x)=\min \left\{x(t)-\widetilde{x}(t), r_{2}\right\}$ and denote

$$
e(x)=\left\{t \in[0,1] \mid x(t)-\widetilde{x}(t)>r_{2}\right\} .
$$

When $x(t)-\widetilde{x}(t)<0, \widetilde{y}(t)=x(t)-\widetilde{x}(t) \geq x(t)-r_{2} \geq-r_{2}$, and so $\|\widetilde{y}\| \leq r_{2}$. Thus for $x \in W$, we have from $(3.6)$

$$
\begin{aligned}
x(t)= & \mu(\widetilde{A} x)(t) \leq \int_{0}^{1} G(t, s) h_{2}(s) g_{2}(x(s)-\widetilde{x}(s)) d s+\widetilde{x}(t) \\
= & \int_{e(x)} G(t, s) h_{2}(s) g_{2}(x(s)-\widetilde{x}(s)) d s \\
& +\int_{[0,1] \backslash e(x)} G(t, s) h_{2}(s) g_{2}(x(s)-\widetilde{x}(s)) d s+\widetilde{x}(t) \\
\leq & \sigma \widetilde{\lambda_{1}} \int_{0}^{1} G(t, s) h_{2}(s) x(s) d s+\int_{0}^{1} G(t, s) h_{2}(s) g_{2}(\widetilde{y}(s)) d s+2 \widetilde{x}(t) \\
\leq & \sigma \widetilde{\lambda_{1}} \int_{0}^{1} G(t, s) h_{2}(s) x(s) d s+M=\left(\widetilde{T}_{2} x\right)(t)+M,
\end{aligned}
$$

where $M$ is defined as 3.7). Thus $\left(\left(I-\widetilde{T}_{2}\right) x\right)(t) \leq M, t \in[0,1]$. As in [8] we can prove that the inverse operator $\left(I-\widetilde{T}_{2}\right)^{-1}$ exists and

$$
\left(I-\widetilde{T}_{2}\right)^{-1}=I+\widetilde{T}_{2}+\widetilde{T}_{2}^{2}+\cdots+\widetilde{T}_{2}^{n}+\cdots .
$$

It follows from $\widetilde{T}_{2}(P) \subset P$ that $\left(I-\widetilde{T}_{2}\right)^{-1}(P) \subset P$. So we have $x(t) \leq(I-$ $\left.\widetilde{T}_{2}\right)^{-1} M, t \in[0,1]$ and $W$ is bounded.

Select $r_{3}>\max \left\{r_{2}, \sup W+\|\widetilde{x}\|\right\}$ and thus $\widetilde{A}$ has no fixed point on $\partial B_{r_{3}}$. In fact, if there exists $x_{2} \in \partial B_{r_{3}}$ such that $\widetilde{A} x_{2}=x_{2}$, then $x_{2} \in W$ and $\left\|x_{2}\right\|=r_{3}>$ $\sup W$, which is a contradiction. Then we have from the permanence property and the homotopy invariance property of fixed point index that

$$
\operatorname{deg}\left(I-\widetilde{A}, B_{r_{3}}, \theta\right)=i\left(\widetilde{A}, B_{r_{3}} \cap P, P\right)=i\left(\theta, B_{r_{3}} \cap P, P\right)=1 .
$$

Set the completely continuous homotopy $H(\lambda, x)=A(x-\lambda \widetilde{x})+\lambda \widetilde{x},(\lambda, x) \in$ $[0,1] \times \bar{B}_{r_{3}}$. If there exists $\left(\lambda_{0}, x_{3}\right) \in[0,1] \times \partial B_{r_{3}}$ such that $H\left(\lambda_{0}, x_{3}\right)=x_{3}$, then $A\left(x_{3}-\lambda_{0} \widetilde{x}\right)=x_{3}-\lambda_{0} \widetilde{x}$ and $\widetilde{A}\left(x_{3}-\lambda_{0} \widetilde{x}+\widetilde{x}\right)=x_{3}-\lambda_{0} \widetilde{x}+\widetilde{x}$. Thus $x_{3}-\lambda_{0} \widetilde{x}+\widetilde{x} \in W$ and

$$
\left\|x_{3}-\lambda_{0} \widetilde{x}+\widetilde{x}\right\| \geq\left\|x_{3}\right\|-\left(1-\lambda_{0}\right)\|\widetilde{x}\| \geq r_{3}-\|\widetilde{x}\|>\sup W
$$

a contradiction! From the homotopy invariance of topological degree and (3.8) we have

$$
\begin{aligned}
\operatorname{deg}\left(I-A, B_{r_{3}}, \theta\right) & =\operatorname{deg}\left(I-H(0, \cdot), B_{r_{3}}, \theta\right) \\
& =\operatorname{deg}\left(I-H(1, \cdot), B_{r_{3}}, \theta\right)=\operatorname{deg}\left(I-\widetilde{A}, B_{r_{3}}, \theta\right)=1 .
\end{aligned}
$$

By (3.5) and (3.9) we have that

$$
\operatorname{deg}\left(I-A, B_{r_{3}} \backslash \bar{B}_{r_{1}}, \theta\right)=\operatorname{deg}\left(I-A, B_{r_{3}}, \theta\right)-\operatorname{deg}\left(I-A, B_{r_{1}}, \theta\right)=1,
$$


which implies that $A$ has at least one fixed point on $B_{r_{3}} \backslash \bar{B}_{r_{1}}$. This means that the singular nonlinear boundary value problem (1.1) has at least one nontrivial solution.

Corollary 3.2. Suppose that the conditions $(H 1)-(H 3)$ are satisfied. If there exists a constant $b^{*} \geq 0$ such that

$$
f(t, x) \geq-\beta^{2} x-\frac{b^{*}}{\widetilde{M}}, \quad \forall t \in[0,1], x \geq-b^{*},
$$

where $\widetilde{M}=\max _{t \in[0,1]} \int_{0}^{1} G(t, s) d s$ and in addition, (3.2) and $(3.3)$ hold, then the singular boundary value problem (1.1) has at least one nontrivial solution.

Proof. Denote

$$
g_{1}(t, x)= \begin{cases}f(t, x)+\beta^{2} x, & t \in[0,1], x \geq-b^{*} \\ f\left(t,-b^{*}\right)+\beta^{2} x, & t \in[0,1], x<-b^{*}\end{cases}
$$

Define

$$
\left(A_{1} x\right)(t)=\int_{0}^{1} G(t, s) g_{1}(s, x(s)) d s, \quad t \in[0,1] .
$$

By Theorem 3.1 we know that $A_{1}$ has at least one nontrivial fixed point $\widetilde{x}$. Then

$$
\widetilde{x}(t)=\int_{0}^{1} G(t, s) g_{1}(s, \widetilde{x}(s)) d s \geq-\frac{b^{*}}{\widetilde{M}} \int_{0}^{1} G(t, s) d s \geq-b^{*} .
$$

From 3.10 we have that $g_{1}(t, \widetilde{x}(t))=g(t, \widetilde{x}(t)), t \in[0,1]$, then

$$
\widetilde{x}(t)=\int_{0}^{1} G(t, s) g_{1}(s, \widetilde{x}(s)) d s=\int_{0}^{1} G(t, s) g(s, \widetilde{x}(s)) d s .
$$

Thus $\widetilde{x}$ is the nontrivial solution of singular boundary value problem 1.1 .

Remark 3.3. In Theorem 3.1 and Corollary 3.2, we do not assume that $g(t, x) \geq 0$ for $x \geq 0$. And it is difficult to obtain those theorems using the theory of fixed point index on a cone. In order to obtain the existence of nontrivial solution, we make use of topological degree theory which is not confined in a cone.

Acknowledgements: The authors were supported by the National Natural Science Foundation of China (No. 10671167).

\section{REFERENCES}

1. W. Feng, JRL. Webb, Solvability of a three-point nonlinear BVPs at resonance, Nonlinear Anal. 30 (1997), 3227-3238. 1

2. R. Ma, Multiplicity results for a three-point boundary value problems at resonance, Nonlinear Anal. 53 (2003), 777-789. 1

3. Z. Bai, W. Li and W. Ge, Existence and multiplicity of solutions for four-point BVPs at resonance, Nonlinear Anal. 60 (2005), 1551-1562. 1 
4. X. Han, Positive solutions for a three-point boundary value problems at resonance, J. Math. Anal. Appl. 336 (2007), 556-568. 1, 2

5. C. Bai, J. Fang, Existence of positive solutions for three-point boundary value problems at resonance, J. Math. Anal. Appl. 291 (2004), 538-549.

6. G. Infante, M. Zima, Positive solutions of multi-point boundary value problems at resonance, Nonlinear Anal. to appear. 1

7. J. Mawhin, Topological Degree Methods in Nonlinear Boundary Value Problems, Berlin, 1979. 1

8. G. Zhang, J. Sun, Positive solutions of m-point boundary value problems, J. Math. Anal. Appl. 291 (2004), 406-418. 1, 2, 3, 3,

9. D. Guo, V. Lakshmikantham, Nonlinear Problems in Abstract Cones, Academic Press, San Diego, 1988. 1, 2

10. D. Guo, J. Sun, Nonlinear Integral Equations, Shandong Press of Science and Technology, Jinan, 1987 (in Chinese).

11. Deimling Klaus, Nonlinear Functional Analysis, Springer-Verlag, New York, 1985. 1

${ }^{1}$ Department of Mathematics and Physics, Taizhou Teachers College, Taizhou, 225300 , CHINA.

E-mail address: xiaorongwu188@163.com

2 School of Mathematics and Physics, Jiangsu Polytechnic University, Changzhou, 213164, China.

E-mail address: fengwang188@163.com 